\title{
Noise-Cancelling CMOS Active Inductor and Its Application in RF Band-Pass Filter Design
}

\author{
Santosh Vema Krishnamurthy, Kamal El-Sankary, and Ezz El-Masry
}

Sexton Campus, Dalhousie University, 1360 Barrington Street, Halifax, Canada NS B3J 1Z1

Correspondence should be addressed to Kamal El-Sankary, kamal.el-sankary@dal.ca

Received 6 August 2009; Revised 21 October 2009; Accepted 4 January 2010

Academic Editor: Liang-Hung Lu

Copyright (C) 2010 Santosh Vema Krishnamurthy et al. This is an open access article distributed under the Creative Commons Attribution License, which permits unrestricted use, distribution, and reproduction in any medium, provided the original work is properly cited.

\begin{abstract}
A CMOS active inductor with thermal noise cancelling is proposed. The noise of the transistor in the feed-forward stage of the proposed architecture is cancelled by using a feedback stage with a degeneration resistor to reduce the noise contribution to the input. Simulation results using $90 \mathrm{~nm}$ CMOS process show that noise reduction by $80 \%$ has been achieved. The maximum resonant frequency and the quality factor obtained are $3.8 \mathrm{GHz}$ and 405 , respectively. An RF band-pass filter has been designed based on the proposed noise cancelling active inductor. Tuned at $3.46 \mathrm{GHz}$, the filter features total power consumption of $1.4 \mathrm{~mW}$, low noise figure of $5 \mathrm{~dB}$, and IIP3 of $-10.29 \mathrm{dBm}$.
\end{abstract}

\section{Introduction}

Inductors, either passive or simulated by active devices are key components in Radio Frequency Integrated Circuits (RFIC) analog building blocks such as filters [1], oscillators [2], phase shifters [3], and low-noise amplifiers (LNA) [4]. As integrated circuit technologies are progressing, the usage of passive inductors is degrading due to their large chip area, low-quality factor, and less tunability. Mainly the inductor is the major chip area consuming building block [5]; the higher the inductance required, the higher the chip area. While tunability can be implemented using passive inductor and low-resistance switches, continuous tuning is not easily achieved. Due to these disadvantages, the concept of active inductors is becoming more attractive.

Many proposed active inductor architectures using MOS transistors [1-5] can be found in the literature. Higher noise, nonlinearity and power consumption are the major disadvantages of active inductors due to the fact that these circuits are realized using active devices which have higher noise. Thermal noise cancelling (NC) has been successfully implemented in LNA circuits by cancelling the noise of their input transistors $[6,7]$. In this paper, $\mathrm{NC}$ is applied to active inductor architecture. First the transistor contributing most of the noise of the active inductor is identified in the forward path of Gyrator-C structure, and its noise contribution is cancelled using the feedback path while the noise contribution of the latter to the input is degenerated by the inclusion of shielding resistor in the feedback. NC allows active inductors to be used in applications such as front-end active RF filter in receivers and also to design low-phase noise oscillators.

The paper is organised as follows. In Section 2, the NC active inductor is presented, as well as the principle of NC and simulation results. In Section 3, a differential secondorder RF band-pass filter based on the proposed NC active inductor and its performance are demonstrated. Finally, the conclusion is given in Section 4 .

\section{NC Active Inductor Design}

In the active inductor circuit shown in Figure 1(a), $M_{2}$ is identified as the transistor with the highest noise contribution.

2.1. Noise Contribution of the Transistor $M_{2}$. The circuit used to implement NC in active inductor is the GyratorC structure proposed in [1] and shown in Figure 1(a). In order to apply NC, we studied the noise contribution of each 


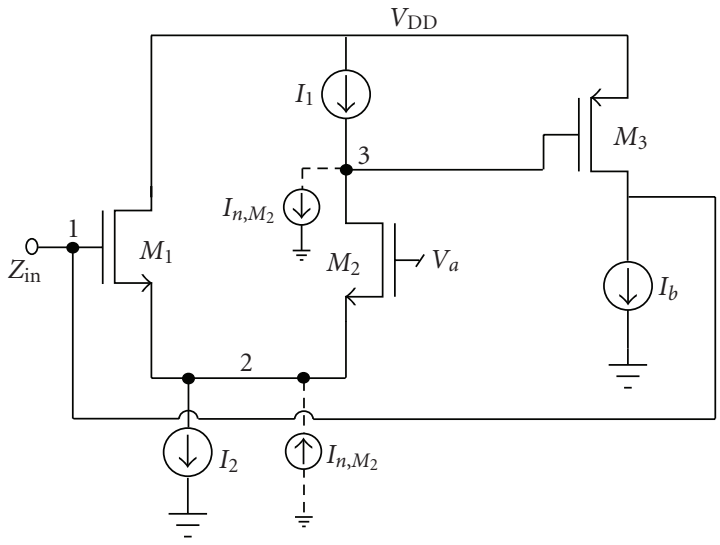

(a)

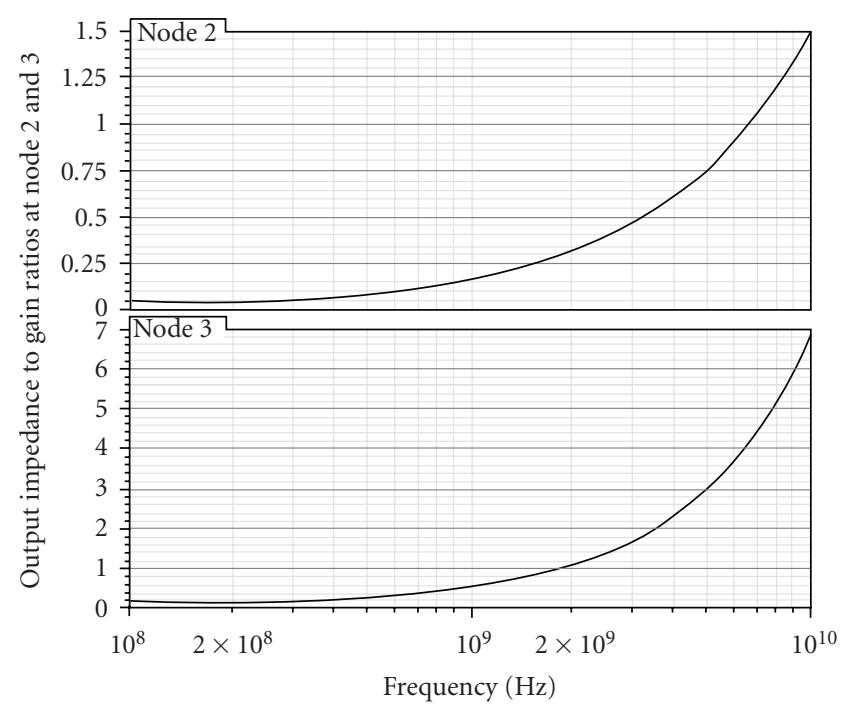

(b)

Figure 1: (a) Active Inductor Circuit without NC. (b) The ratio of output impedance to the respective gain at nodes 2 and 3.

transistor and identified the transistor that has the highest percentage of noise to the total input referred noise. Usually in LNA, noise cancelling is applied to the input transistor. This is not the case of active inductors, and due to the backto-back $G_{m}$ structure a careful study is needed to identify the transistor that contributes the highest noise at the frequency of interest.

The noise current of $M_{2}$ is modelled as the current source $I_{n, M_{2}}$, between nodes 2 and 3 in Figure 1(a). For better understanding, we have shown the noise of $M_{2}$ as two current sources, one flowing out of node 3 and other flowing into node 2 [8]. From noise analysis and simulation results, $M_{2}$ is found to be contributing more than $25 \%$ to the input noise at high frequency. The reason behind this high noise contribution is that the noise of $M_{2}$ will be amplified by the gains from the nodes 2 and 3 when referred to the input. These gains depend on the impedance at nodes 2 and 3 divided by the respective gains calculated from input node 1 to the nodes 2 and 3. As shown in Figure 1(b), the output impedance to the gain ratios go higher as frequency increases. Thus the noise current of $M_{2}$ multiplied by these two ratios to be referred to the input goes higher as frequency increases.

The input referred noise from nodes 2 and 3 can be calculated by breaking the feedback from drain of $M_{3}$ to the input. The input referred noise contribution from node 3 is calculated as

$$
\begin{aligned}
I_{n, M 2 \mathrm{ref}_{\mathrm{node}}} & =\frac{I_{n, M 2_{\text {node }}} R_{03 \mathrm{CL}}}{A_{I 3 \mathrm{CL}}} \\
& =I_{n, M 2_{\text {node }}} \frac{R_{03 \mathrm{OL}} /\left(1+A_{I 3 \mathrm{OL}} \beta\right)}{A_{I 3 \mathrm{OL}} /\left(1+A_{I 3 \mathrm{OL}} \beta\right)} \\
& =\frac{I_{n, M 2_{\text {node }}} R_{03 \mathrm{OL}}}{A_{I 3 \mathrm{OL}}} .
\end{aligned}
$$

Hence,

$$
I_{n, M 2 \mathrm{ref}_{\text {node3 }}}=I_{n, M 2_{\text {node } 3}} \frac{C_{2} s+\left(g_{m 1}+g_{m 2}+1 / r_{02}\right)}{g_{m 1} g_{m 2} c_{1} s+g_{m 1} g_{m 2}} .
$$

Similarly, we can obtain the input referred noise from node 2 as

$$
I_{n, M 2 \mathrm{ref}_{\text {node } 2}}=\frac{I_{n, M 2_{\text {node2 }}}\left(C_{2} s+r_{o 2} r_{o b}\left(g_{m 1}+g_{m 2}+1 / r_{o 2}\right)\right)}{g_{m 1}\left(1+c_{1} s\right)\left(1+r_{o b} / r_{o 2}+r_{o b} c_{3} s+\left(g_{m 1}+g_{m 2}\right) r_{o b}\right)},
$$

where $I_{n, M 2 \mathrm{ref}_{\text {node2,3 }}}$ are the input referred noise currents of $M_{2}$ from nodes 2 and 3 , respectively. $I_{n, M 2_{\text {node2,3 }}}$ are noise currents of $M_{2}$ at nodes 2 and 3, $R_{03 \mathrm{CL}}$ is the closed loop resistance at node $3, R_{03 \mathrm{OL}}$ is the open loop resistance at node $3, A_{I 3 \mathrm{CL}}$ is the closed loop transimpedance gain from node 1 to node 3 for the shunt-shunt feedback circuit in Figure 1(a), $A_{I 3 \mathrm{OL}}$ is the open loop transimpedance gain from node 1 to node 3 , and $\beta=g_{m 3}$ is the feedback factor.

Equations (2) and (3) reveal that the noise contribution of $M_{2}$ goes higher at high frequencies. This can be understood from the fact that the Gyrator structure in Figure 1(a) has shunt-shunt feedback structure and the capability of this feedback topology to lower the output impedances at nodes 2 and 3 is reduced at higher frequencies due to the feedback forward gain reduction. That leads to higher contribution of the noise sources $I_{n, M 2_{\text {node2 }, 3}}$ when multiplied by these output impedances at nodes 2 and 3 to be referred to the input at node 1.

2.2. NC Principle and Simulation Results. Figure 2 illustrates the modification introduced for cancelling the noise of $M_{2}$ ( $R_{f}$ is neglected at this time and nodes 1 and 4 are shorted). The noise current of $M_{2}$, which is modelled as the current source $I_{n, M 2}$, flows into node 2 but out of node 3 as shown 


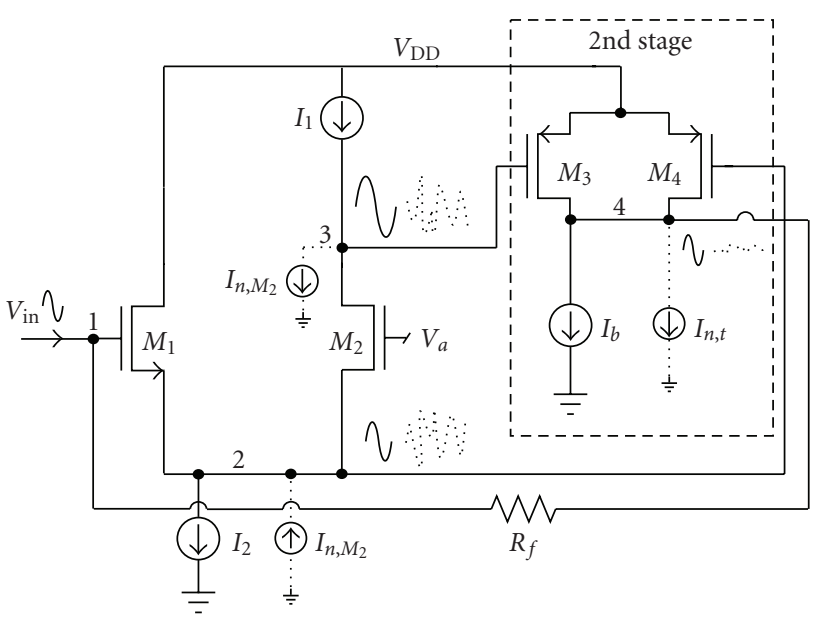

Figure 2: Principle of noise cancelling design.

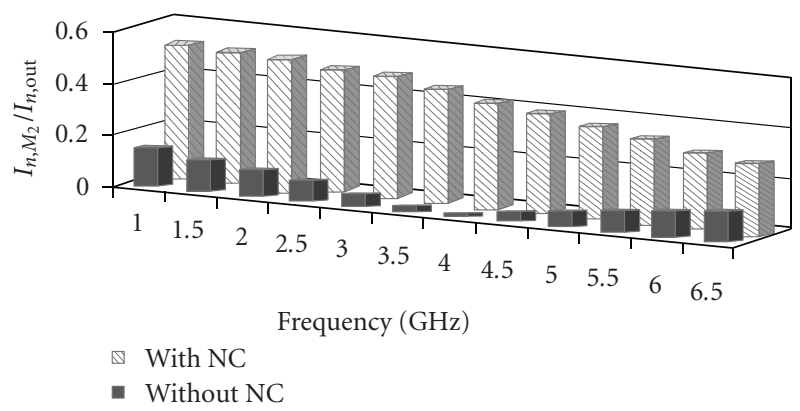

FigURE 3: Simulated $I_{n, M 2} / I_{n, \text { out }}$ versus frequency with and without NC.

in Figure 2. This creates two fully correlated noise voltages at nodes 2 and 3 with opposite phases. These two voltages are converted into current by $M_{3}$ and $M_{4}$, respectively. By properly matching the AC currents of $M_{3}$ and $M_{4}$ around the resonant frequency, the noise contributed by $M_{2}$ can be cancelled at the input.

Conversely, the signal voltages at nodes 2 and 3 are in phase, resulting in constructive addition at node 1 . The condition for complete noise cancelling of $M_{2}$ can be derived as

$$
\begin{aligned}
& I_{n, \text { input }}=I_{n, M 2} Z_{O 3} g_{m 3}-I_{n, M 2} Z_{O 2} g_{m 4}=0 \\
& \Longrightarrow \frac{g_{m 2} r_{o 2}+g_{m 1} r_{o 2}+C_{2} r_{o 2} s}{s^{2} C_{2} c_{3} r_{o 2}+s c_{3} r_{o 2}\left(g_{m 2}+g_{m 1}\right)+r_{o 2}\left(g_{m 2}+g_{m 1}\right)+g_{m 1}} g_{m 3} \\
& =\frac{r_{o 2} r_{o b}}{r_{o 2} r_{o b} g_{m 1}+r_{o 2} r_{o b} g_{m 2}+s C_{2} r_{o 2} r_{o b}+r_{o 2}+r_{o b}} g_{m 4},
\end{aligned}
$$

where $Z_{O 2,3}$ are the total impedance at the nodes 2 and $3, r_{03,4, b}$ are the output resistances of transistors $M_{3,4, b}$, and $C_{1,2,3}$ are the total capacitances at nodes 1,2 , and 3.

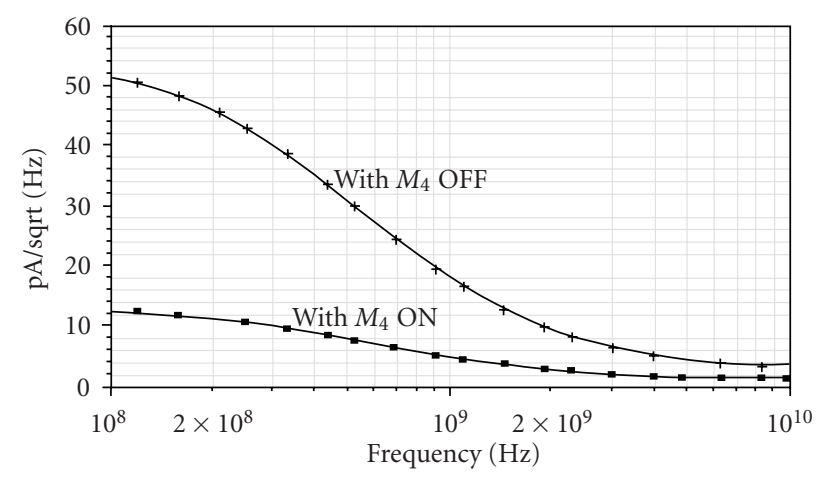

FIGURE 4: Simulated input referred noise with and without NC.

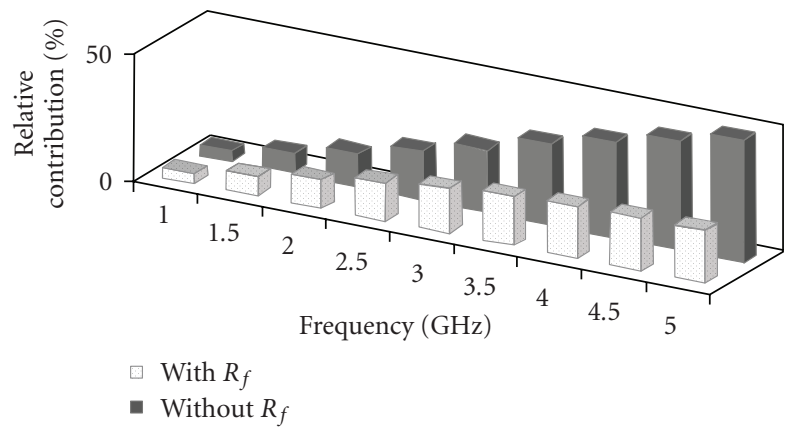

FigURE 5: Simulated noise contribution with and without $R_{f}$.

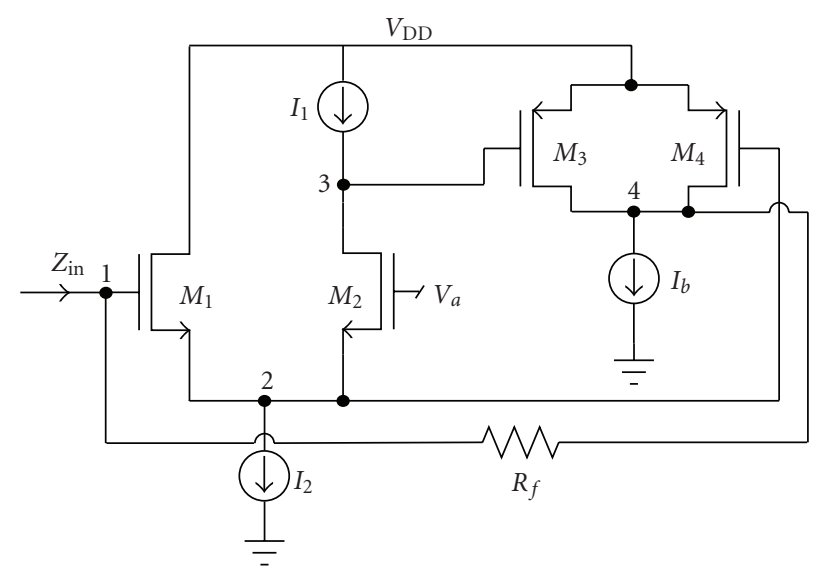

Figure 6: Simplified active inductor.

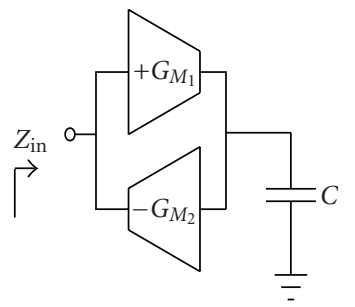

(a)

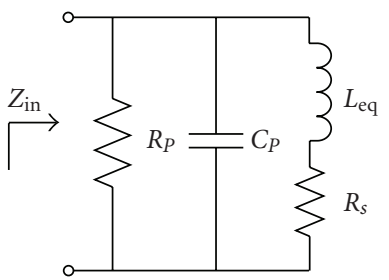

(b)
Figure 7: (a) Gyrator-C realization of active inductor (b) equivalent passive model. 


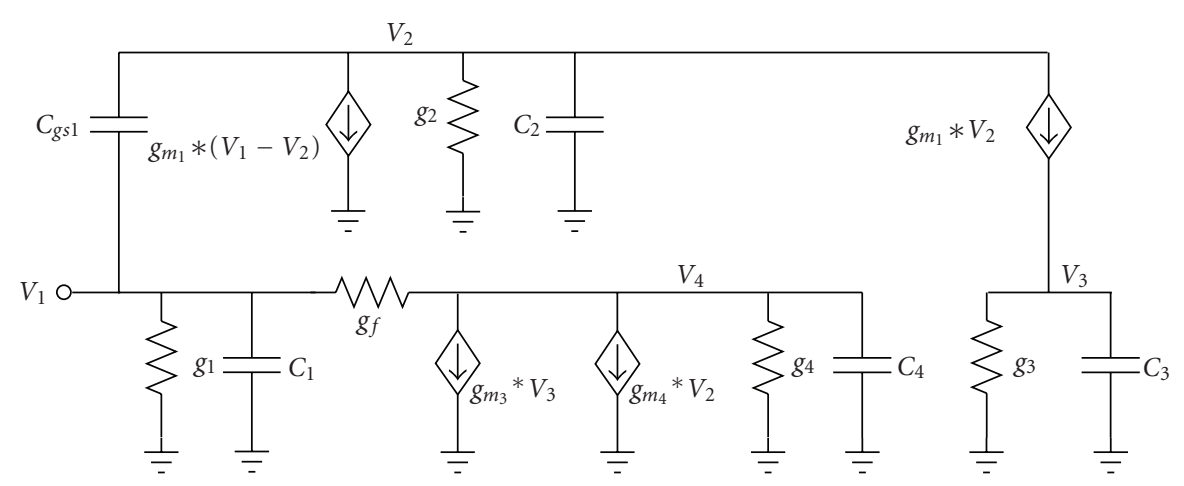

FIGURE 8: Small signal model of the simplified active inductor in Figure 1.

For simplicity, we have ignored few terms in the above $\mathrm{NC}$ condition in (5). Figure 3 compares the noise contribution of $M_{2}$ with and without NC to the total output noise after the above NC condition is matched relatively at the frequencies of interest by properly choosing the size and biasing current of $M_{3}$ and $M_{4}$. We can see from Figure 3 that the noise contribution from $M_{2}$ is reduced at all frequencies and it is almost contributing $0 \%$ around the resonance frequency.

To further confirm the effect of noise cancelling, the case with $M_{4}$ being turned OFF is simulated while maintaining the same power consumption by the following steps: (1) connecting the gate of $M_{4}$ to $V_{\mathrm{DD}}$; (2) $V_{a}$ is adjusted accordingly to increase the bias current of $M_{2}$; (3) the biasing current of $M_{3}$ is adjusted to give the same power dissipation as $M_{4}$ is turned $\mathrm{ON}$. The simulated total input referred noise current when $M_{4}$ is turned ON/OFF is shown in Figure 4. It should be noticed from this graph that the noise has been reduced by $80 \%$ when $M_{4}$ is ON (with NC).

Even after cancelling the noise of $M_{2}$, we can still further suppress the noise of the second stage in Figure 2. We can see that the entire noise of the second stage is directly added to the input noise as is the case in every back-to-back Gyrator active inductor. To further optimize the active inductor for lower noise, the resistor $R_{f}$ is added to degenerate the noise $i_{n, t}$ of the entire 2 nd stage as shown in Figure 2. Even though $R_{f}$ will introduce noise to the input but the benefit of its inclusion on the noise reduction overshadows the effect of its added noise. The noise contributed by the second stage to the input while $R_{f}$ is included can be calculated as below. Figure 2 can be considered as shunt-shunt feedback structure where $M_{1-4}$ are considered to be in feed-forward loop and $R_{f}$ in the feedback loop.

The noise contribution of the second stage can be obtained as

$$
\begin{aligned}
I_{n, \text { input }} & =\frac{I_{n, t} Z_{o 4 \mathrm{CL}}}{A_{I 4 \mathrm{CL}}} \\
& =I_{n, t} \frac{Z_{o 4 \mathrm{OL}} /\left(1+A_{I 4 \mathrm{OL}} \beta\right)}{A_{I 4 \mathrm{OL}} /\left(1+A_{I 4 \mathrm{OL}} \beta\right)} \\
& =\frac{I_{n, t} Z_{o 4 \mathrm{OL}}}{A_{I 4 \mathrm{OL}}},
\end{aligned}
$$

$$
I_{n, \text { input }}=\frac{I_{n, t} g_{m 2} r_{o 2}}{R_{f} A_{1}\left(g_{m 2} g_{m 3} r_{o 2}+g_{m 4}\right)} \approx \frac{I_{n, t}}{R_{f} A_{1} g_{m 3}}
$$

where $I_{n, t}$ is the total noise current of the entire 2nd stage, $Z_{o 4 \mathrm{CL}}, A_{I 4 \mathrm{CL}}$ are the closed loop impedance and gain at node $4, Z_{04 \mathrm{OL}}, A_{\text {I4OL }}$ are the open loop impedance and gain at node 4 and $A_{1}$ is the gain contributed from $M_{1}$ and $M_{2}$. From (7), it is evident that increasing $R_{f}$ shields the input node of the active inductor from the noise contribution of the second stage formed by $M_{3}, M_{4}$, and $I_{b}$. Figure 5 shows the simulated noise contribution of the second stage when compared with and without $R_{f}$. Figure 5 shows the noise contribution of second stage to the total input referred noise. Here we can see that by adding $R_{f}$, the noise contribution of 2 nd stage has been reduced to $15 \%$ from $45 \%$.

2.3. Active Inductor Parameters. In this section, the different parameters of the proposed active inductor are derived. The simplified active inductor is shown in Figure 6. The Gyrator$\mathrm{C}$ principle on which active inductor is based on is depicted in Figure 7(a) and its equivalent passive model is shown in Figure 7(b). The differential pair $M_{1}$ and $M_{2}$ forms the positive transconductance $G_{m 1}$ with input node at 1 and output nodes at 2 and 3. The transistor pair $M_{3}$ and $M_{4}$ forms the negative transconductance $-G_{m 2}$ with input nodes at 2 and 3 and output node at 1 . These two $G_{m}$ blocks convert the parasitic capacitors at nodes 2 and 3 to an equivalent inductor appearing at node 1 . The ideal current sources in the active inductor are implemented using simple current mirror.

The equivalent $Z_{\text {in }}$ can be obtained from the small signal analysis circuit in Figure 8. For better intuition, a few parasitic parameters have been ignored. The approximate expression for $Z_{\text {in }}$ is as follows:

$$
Z_{\text {in }}(s) \approx \frac{s / C_{1}+g_{3} / C_{1} C_{3}}{s^{2}+s \mathfrak{A}+\mathfrak{B}},
$$

where $\mathfrak{A}$ denotes $\left(g_{m 4} g_{m 1} / G_{m} C_{1}\right)\left(g_{4} g_{f} /\left(g_{4}+g_{f}\right)\right)+g_{1} / C_{1}+$ $\left.g_{3} / C_{3}+g_{1} g_{3} C_{2} / G_{m} C_{1} C_{3}-\omega^{2}\left(C_{2} / G_{m}\right)\right)$ and $\mathfrak{B}$ denotes $\left(\left(g_{m 1} g_{m 2} g_{m 3}+g_{m 1} g_{m 4} g_{3}\right) / G_{m} C_{1} C_{3}\right)\left(g_{f} /\left(g_{4}+g_{f}\right)\right.$, and $g_{m 1-4}$ are 


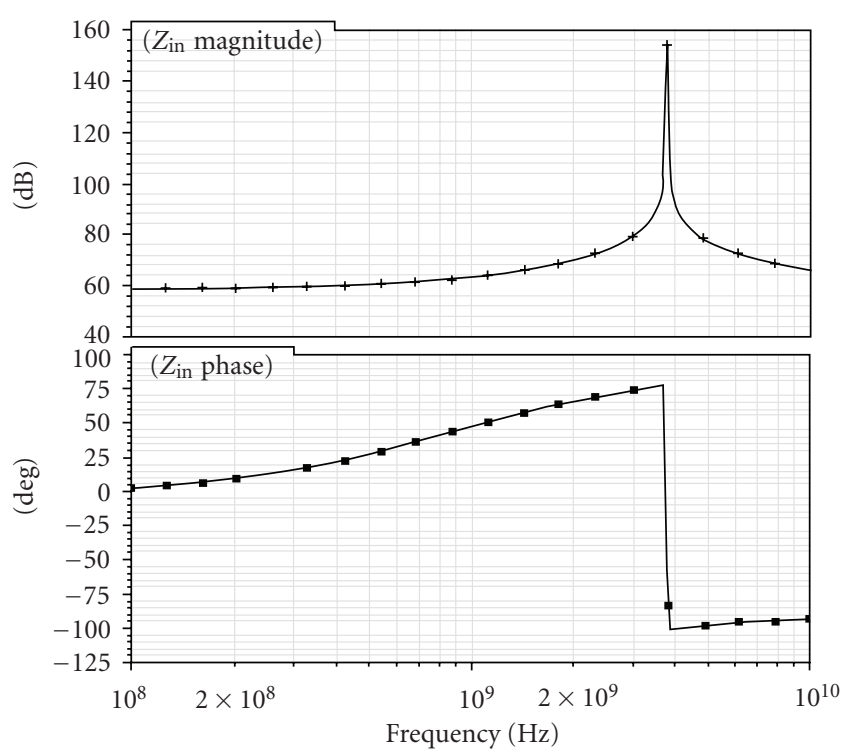

FIGURE 9: Simulated frequency response.

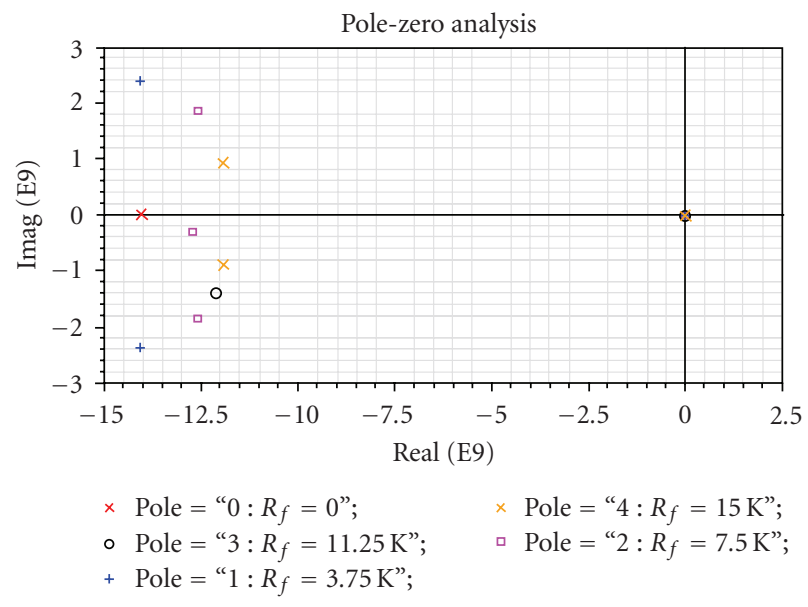

FIgURE 10: Pole-zero plot for variation of $R_{f}$.

the transconductances of transistors $M_{1-4}, C_{1-3}, g_{1-4}$ are the total parasitic capacitances and conductances at nodes $1-4$, $g_{f}$ is the conductance of feedback resistor, $G_{m}=g_{m 1}+g_{m 2}+g_{3}$, and the cubic term in the denominator is substituted to $-\omega^{2} s$, because the effect of negative real pole arising due to cubic term is of second order [1] when compared to dominant conjugate poles.

From (8), $Z_{\text {in }}$ is equivalent to the input impedance of the RLC passive network shown in Figure 7(b). The passive equivalent elements are obtained as

$$
R_{s}=\frac{G_{m} g_{3}}{\left(g_{m 1} g_{m 2} g_{m 3}+g_{m 1} g_{m 4} g_{3}\right)\left(g_{f} /\left(g_{4}+g_{f}\right)\right)},
$$

where $R_{s}$ is the series resistor with $L_{\text {eq }}$ and the parallel parasitic capacitor $C_{p}$ are given by

$$
\begin{gathered}
L_{\mathrm{eq}}=\frac{G_{m} C_{3}\left(\left(g_{f}+g_{4}\right) / g_{f}\right)}{g_{m 1} g_{m 2} g_{m 3}+g_{m 1} g_{m 4} g_{3}}, \\
C_{p}=C_{1} .
\end{gathered}
$$

The resonant frequency is given by

$$
\begin{gathered}
\omega_{0}=\sqrt{\frac{g_{m 1} g_{m 2} g_{m 3}+g_{m 1} g_{m 4} g_{3}}{G_{m} C_{1} C_{3}\left(\left(g_{f}+g_{4}\right) / g_{f}\right)}}, \\
f_{0}=\frac{1}{2 \pi \sqrt{L_{\mathrm{eq}} C_{1}}} .
\end{gathered}
$$

The quality factor at the resonant frequency is obtained as

$$
Q_{0}=\frac{\sqrt{\mathfrak{B}}}{\mathfrak{A}} .
$$

The simulated frequency response of the active inductor is shown in Figure 9. The simulations are carried out in $90 \mathrm{~nm}$ STM CMOS process. The transistor sizes (W/L in $\mu \mathrm{m}$ ) are $M_{1}(6 / 0.18), M_{2}(6 / 0.18), M_{3}(11.5 / 0.18), M_{4}(2.3 / 0.18)$, $I_{1}=280 \mu \mathrm{A}, I_{b}=I_{2}=400 \mu \mathrm{A}, V_{a}=0.8 \mathrm{~V}$, and $V_{\mathrm{DD}}=$ $1.2 \mathrm{~V}$. The active inductor resonates at centre frequency of $f_{0}=3.8 \mathrm{GHz}$ with a quality factor of $Q_{0}=405$. The pole-zero analysis performed using Spectre simulator shows that all the poles reside on the left side of the $j \omega$ axis and consequently the active inductor is stable. Also varying the feedback resistor $R_{f}$ (from 0 to $30 \mathrm{k} \Omega$ ) and $g_{m 1}$ and $g_{m 2}$ (by varying the active inductor biasing currents $I_{2}$ and $I_{b}$ from $300 \mu \mathrm{A}$ to $500 \mu \mathrm{A}$ ) was applied to confirm the stability of the active inductor in case of tuning its center frequency and quality factor. Figure 10 shows the zero-pole plot for the active inductor transfer function while changing $R_{f}$ from 0 to $15 \mathrm{k} \Omega$. Moreover, transient analysis has been performed to confirm the stability.

Table 1 compares the performance of the proposed active inductor with the previously published active inductors $[1,9$, 10]. Comparison of performances proves that this inductor has a good quality factor, wide inductive bandwidth, lowpower consumption of $1.2 \mathrm{~mW}$, and very less noise when integrated over $500 \mathrm{MHz}$ bandwidth.

\section{RF Band-Pass Filter and Simulation Results}

Figure 11 shows the proposed pseudo-differential RF Bandpass filter implemented using proposed NC active inductor. The input stage is designed by the common-gate transistor $M_{\text {in }}$ which is biased by $I_{\text {in }}$ and $R_{\text {in }}$. A source follower stage is used as an output buffer for output matching and to reduce the loading effect.

The effect of NC is high lightened by comparing the performance of the two band-pass filters. One designed using the active inductor with $\mathrm{NC}$ and other using the active inductor without NC (the transistor $M_{4}$ is turned OFF as mentioned above). In Figure 12, we can see that when the 


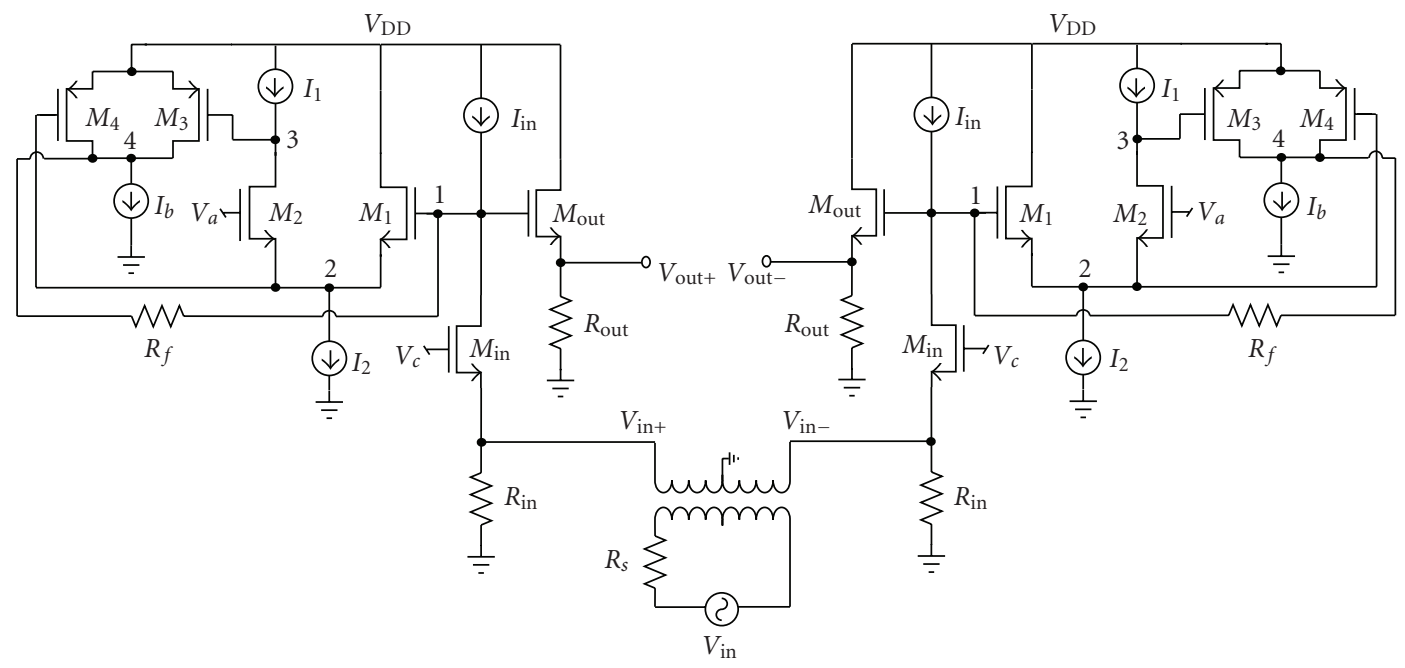

FIGURE 11: RF Band-pass filter based on NC Active inductor.

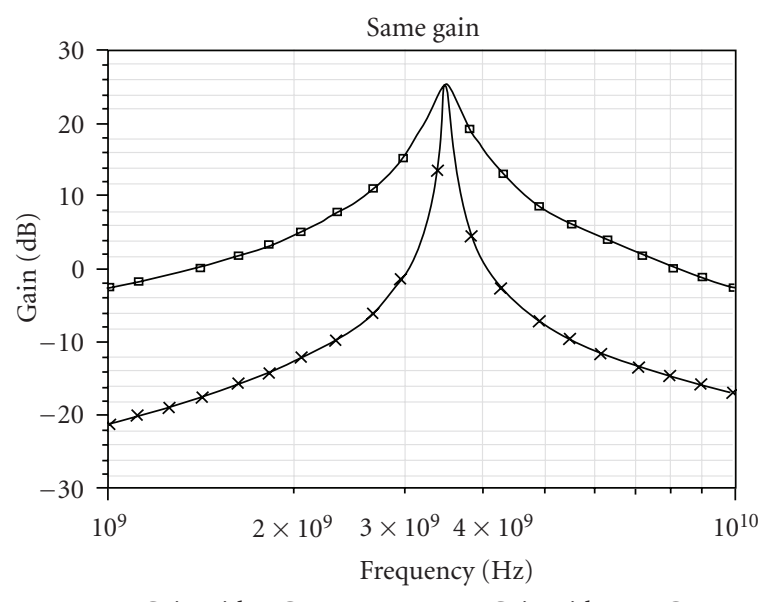

ㅁ Gain with NC

(a)

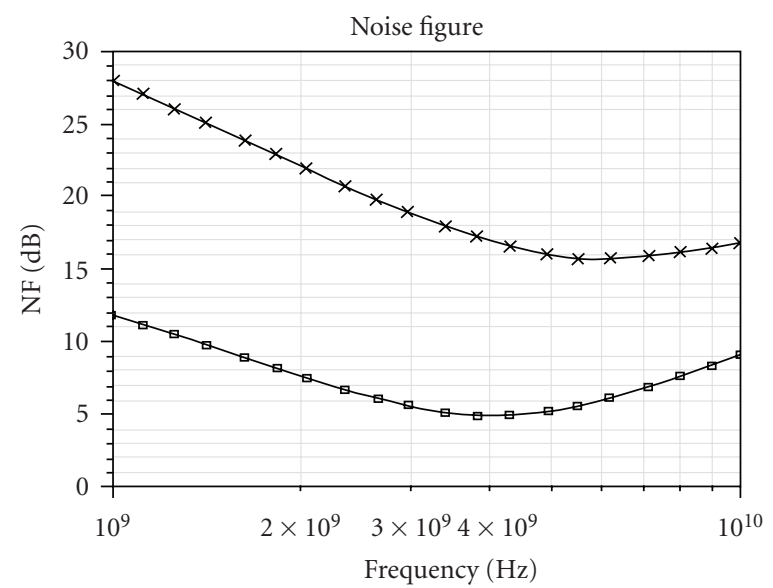

- NF with NC

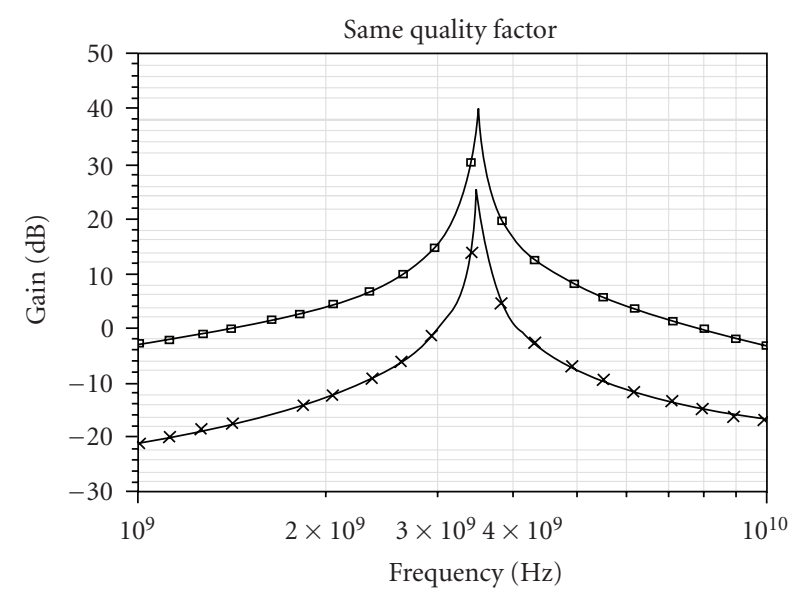

$\times$ Gain without NC

- Gain with NC

(b)

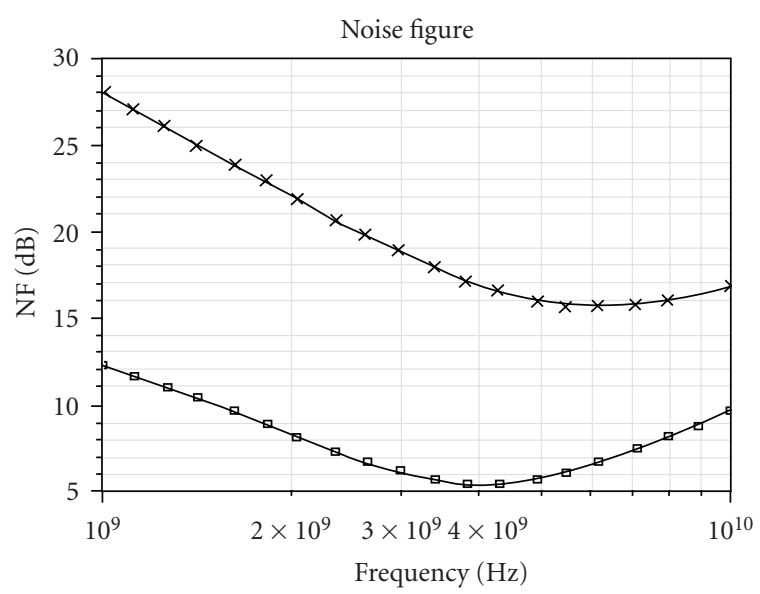

$\times$ NF without NC a NF with NC

(d)

FIGURE 12: NF and gain comparison for band-pass filter with and without NC active inductor. 


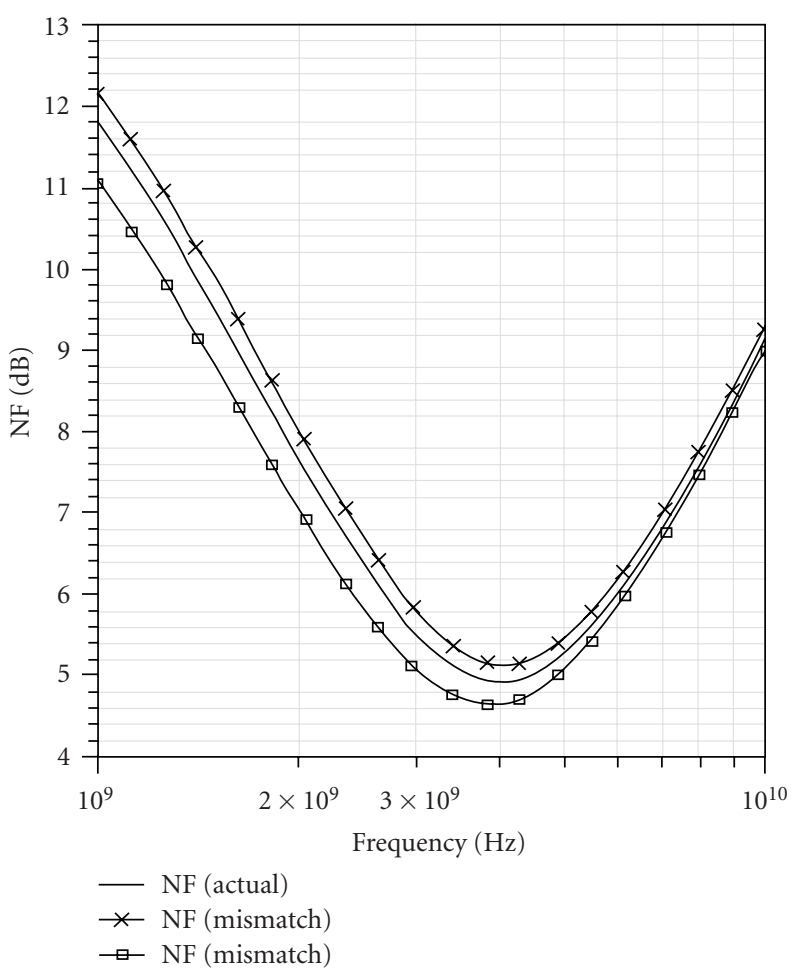

(a)

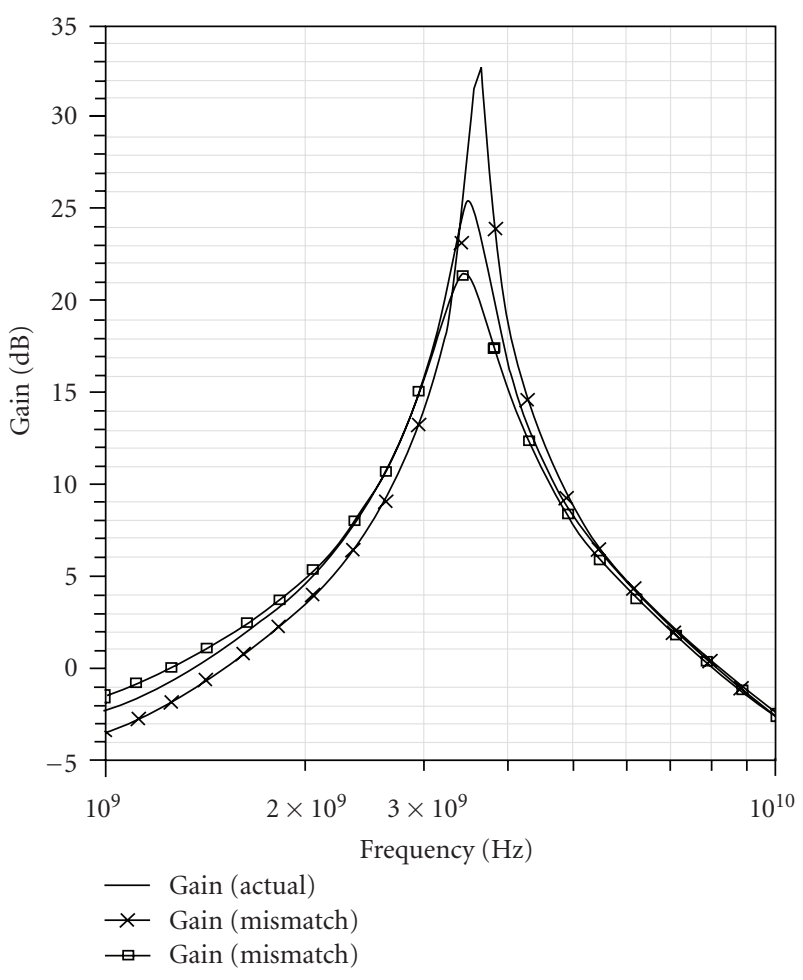

(b)

FIGURE 13: NF and frequency response with process variation.

gain and centre frequency of the proposed band-pass filter are same, the NF without and with $\mathrm{NC}$ are $17 \mathrm{~dB}$ and $5 \mathrm{~dB}$, respectively. And also we can see that when the 2 filters are tuned to have same quality factor and centre frequency, the NF without and with $\mathrm{NC}$ are $17 \mathrm{~dB}$ and $5.7 \mathrm{~dB}$, respectively.

Figure 13 shows the filter NF and frequency response under extreme cases of combined process and mismatch variations. We can see that the NF has changed by almost $0.75 \mathrm{~dB}$ around the centre frequency while the gain of the filter has been changed without much variation in the centre frequency. The gain of the filter can be retrieved by tuning $Q$ using the biasing current $I_{2}$ in Figure 11 .

The linearity of the band-pass filter was examined by plotting the third-order intercept point (IIP3) using periodic steady state simulation as shown in Figure 14. The conditions for simulating the linearity are center frequency $f_{0}=$ $3.467 \mathrm{GHz}$ and the two signals injected were at frequencies of $3.48 \mathrm{GHz}$ and $3.49 \mathrm{GHz}$, respectively. While noise cancelling reduces the noise floor, the harmonic distortion is not reduced as a result of NC conditions, which leads to almost constant dynamic range for the filter with and without NC. However, the harmonic distortion can be cancelled by selecting different conditions other than the one used for NC, therefore, simultaneous cancellation of noise and distortion is hampered [13]. Despite the fact that this filter presents an acceptable linearity, the latter can be improved by increasing the overdrive voltages of transistors $M_{1-4}$ at the cost of higher power supply. Moreover, distortion cancelling techniques

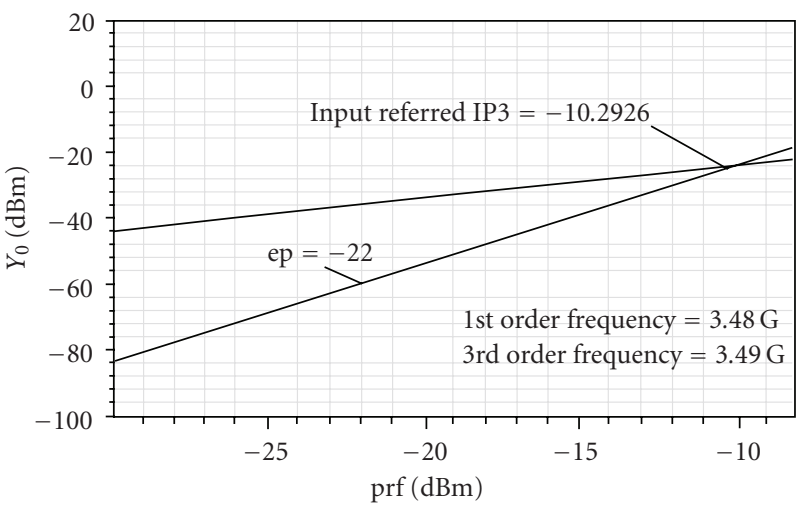

Trace $=$ "3rd order"; ipn curves

Trace $=$ "1st order"; ipn curves

FIGURE 14: IIP3 simulation result.

such as the one presented in [14] can be adapted to cancel the distortion of the proposed active inductor and that to improve the overall linearity of the designed RF filter.

Table 2 compares the performance of the band-pass filter using the proposed active inductor with previously published RF band-pass filters. Comparison of performances proves that this filter features lower-power consumption of $1.4 \mathrm{~mW}$ and lower noise figure of $5 \mathrm{~dB}$ at the filter centre frequency. 
TABLE 1: Comparison of active inductor performances.

\begin{tabular}{lcccc}
\hline Parameter & Reference [1] & Reference [9] & Reference [10] & This work \\
\hline Technology & $0.20 \mu \mathrm{m} / 1.8 \mathrm{~V}$ & $0.13 \mu \mathrm{m} / 1.2 \mathrm{~V}$ & $0.35 \mu \mathrm{m} / 1.5 \mathrm{~V}$ & $90 \mathrm{~nm} / 1.2 \mathrm{~V}$ \\
Inductive bandwidth & n.a. & $300 \mathrm{MHz}-7.32 \mathrm{GHz}$ & $6.8 \mathrm{MHz}-2.97 \mathrm{GHz}$ & $600 \mathrm{MHz}-3.8 \mathrm{GHz}$ \\
$L(\mathrm{nH})$ & 29 & $38-144$ & 30.9 & $165-530$ \\
$Q_{L \max }$ & n.a. & $3900 @ 5.75 \mathrm{GHz}$ & $434 @ 1 \mathrm{GHz}$ & $120 @ 3 \mathrm{GHz}$ \\
$Q_{R}$ & 665 & 61 & n.a. & 405 \\
$P_{\text {dis }}(\mathrm{mW})$ & 4.4 & 1 & 0.6 & 1.2 \\
Noise & $0.8 \mu \mathrm{V} / \sqrt{\mathrm{Hz}}$ & $69.31 \mu \mathrm{V}^{*}$ & $91.3 \mu \mathrm{V}$ & $43.37 \mathrm{nV}$ \\
\hline
\end{tabular}

${ }^{*}$ Calculated.

TABLE 2: Comparison of band-pass filter performances.

\begin{tabular}{lcccc}
\hline Parameter & Reference [1] & Reference [11] & Reference [12] & This work \\
\hline Technology & $0.20 \mu \mathrm{m}$ & $0.18 \mu \mathrm{m}$ & $0.18 \mu \mathrm{m}$ & 5 \\
Filter order & 2 & 2 & $3.45 \sim 3.6$ & $\mathrm{~nm}$ \\
$\omega_{0}(\mathrm{GHz})$ & 5.4 & 2.44 & 28 & 2 \\
$P_{\text {dis }}(\mathrm{mW})$ & 4.4 & 10.8 & 14 & 1.4 \\
NF $(\mathrm{dB})$ & 25.6 & 18 & $($ at $3.6 \mathrm{GHz})$ & 5 \\
& $($ at $5.7 \mathrm{GHz})$ & $-2.45 \mathrm{GHz})$ & -2.4 & $($ at $3.46 \mathrm{GHz})$ \\
IIP3 $(\mathrm{dBm})$ & -13.9 & - & -10.29 \\
\hline
\end{tabular}

\section{Conclusion}

A $3.8 \mathrm{GHz}$ noise cancelling CMOS active inductor is presented. The proposed active inductor has low noise due to noise cancelling and resistive degeneration. The circuit has wide inductive bandwidth, high-resonant frequencies with high-quality factor. Simulation results are provided in $90 \mathrm{~nm}$ 1.2 V STM CMOS process. A second-order band-pass filter has been designed based on this proposed active inductor. The designed filter including the input and output buffer stages has low NF and low-power consumption which makes it suitable for front-end RF filtering applications.

\section{References}

[1] H. Xiao and R. Schaumann, "A $5.4 \mathrm{GHz}$ high-Q tunable active-inductor bandpass filter in standard digital CMOS technology," Analog Integrated Circuits and Signal Processing, vol. 51, no. 1, pp. 1-9, 2007.

[2] H. Xiao and R. Schaumann, "A low-voltage low-power CMOS $5 \mathrm{GHz}$ oscillator based on active inductors," in Proceedings of the 9th International Conference on Electronics Circuits and Systems, vol. 1, pp. 231-234, September 2002.

[3] M. Abdalla, G. V. Eleftheriades, and K. Phang, "A differential $0.13 \mu \mathrm{m}$ CMOS active inductor for high-frequency phase shifters," in Proceedings of the IEEE International Symposium on Circuits and Systems, pp. 3341-3344, 2006.

[4] D. K. Shaeffer and T. H. Lee, "A 1.5 V 1.5 GHz CMOS low noise amplifier," IEEE Journal of Solid-State Circuits, vol. 32, no. 5, pp. 745-759, 1997.

[5] A. Pascht, J. Fischer, and M. Berroth, "A CMOS low noise amplifier at $2.4 \mathrm{GHz}$ with active Inductor load," in Proceedings of the IEEE Topical Meeting on Silicon Monolithic Integrated Circuits in RF Systems, pp. 1-5, 2001.
[6] L. Chih-Fan and L. Shen-luan, "A broadband noise-cancelling CMOS LNA for 3.1-10.6 GHz UMB receivers," IEEE Journal of Solid-State Circuits, vol. 42, no. 2, pp. 329-339, 2007.

[7] B. Federico, A. M. K. Eric, and B. Nauta, "Wide-band CMOS low-noise amplifier exploiting thermal noise cancelling," IEEE Journal of Solid-State Circuits, vol. 39, no. 2, pp. 275-282, 2004.

[8] B. Razavi, Design of Analog CMOS Integrated Circuits, Tata McGraw-Hill, New Delhi, India, 2001.

[9] H. Ugur Uyanik and N. Tarim, "Compact low voltage highQ CMOS active inductor suitable for RF applications," Analog Integrated Circuits and Signal Processing, vol. 51, no. 3, pp. 191194, 2007.

[10] A. Thanachayanont and S. S. Ngow, "Low voltage high-Q VHF CMOS transistor-only active inductor," in Proceedings of the IEEE International Midwest Symposium on Circuits and Systems, vol. 3, pp. 552-555, 2002.

[11] Z. Gao, J. Ma, M. Yu, and Y. Ye, "A fully integrated CMOS active bandpass filter for multiband RF front-ends," IEEE Transactions on Circuits and Systems II, vol. 55, no. 8, pp. 718722, 2008.

[12] K.-H. Liang, C. C. Ho, C.-W. Kuo, and Y.-J. Chan, "CMOS RF band-pass filter design using the high quality active inductor," IEICE Transactions on Electronics, vol. E88-C, no. 12, pp. 23722375, 2005.

[13] F. Bruccoleri, A. M. Klumperink Eric, and B. Nauta, Wideband Low Noise Amplifiers Exploiting Thermal Noise Cancellation, vol. 840 of The Springer International Series in Engineering and Computer Science, Springer, New York, NY, USA, 2005.

[14] W.-H. Chen, G. Liu, B. Zdravko, and A. M. Niknejad, "A highly linear broadband CMOS LNA employing noise and distortion cancellation," IEEE Journal of Solid-State Circuits, vol. 43, no. 5, pp. 1164-1175, 2008. 

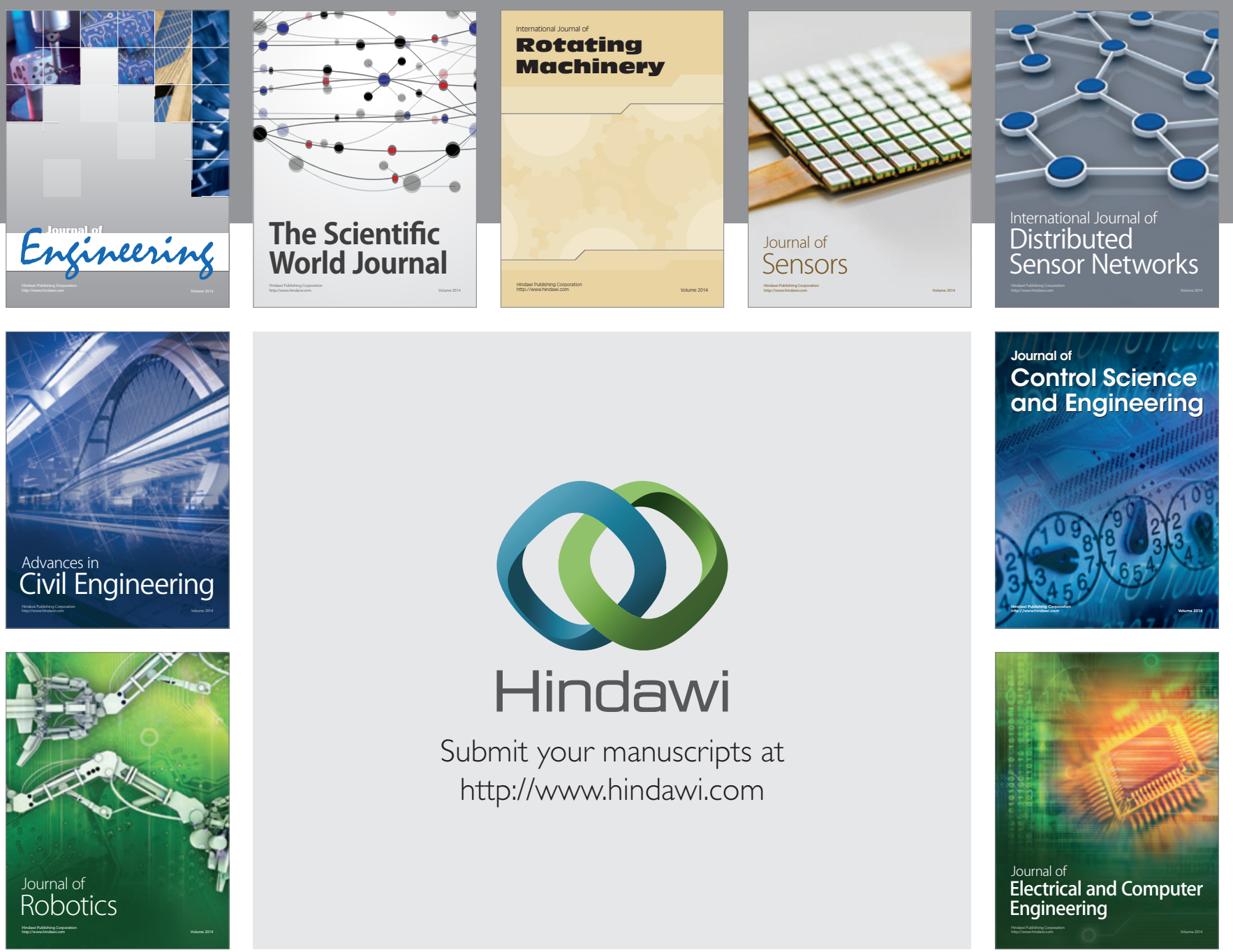

Submit your manuscripts at

http://www.hindawi.com
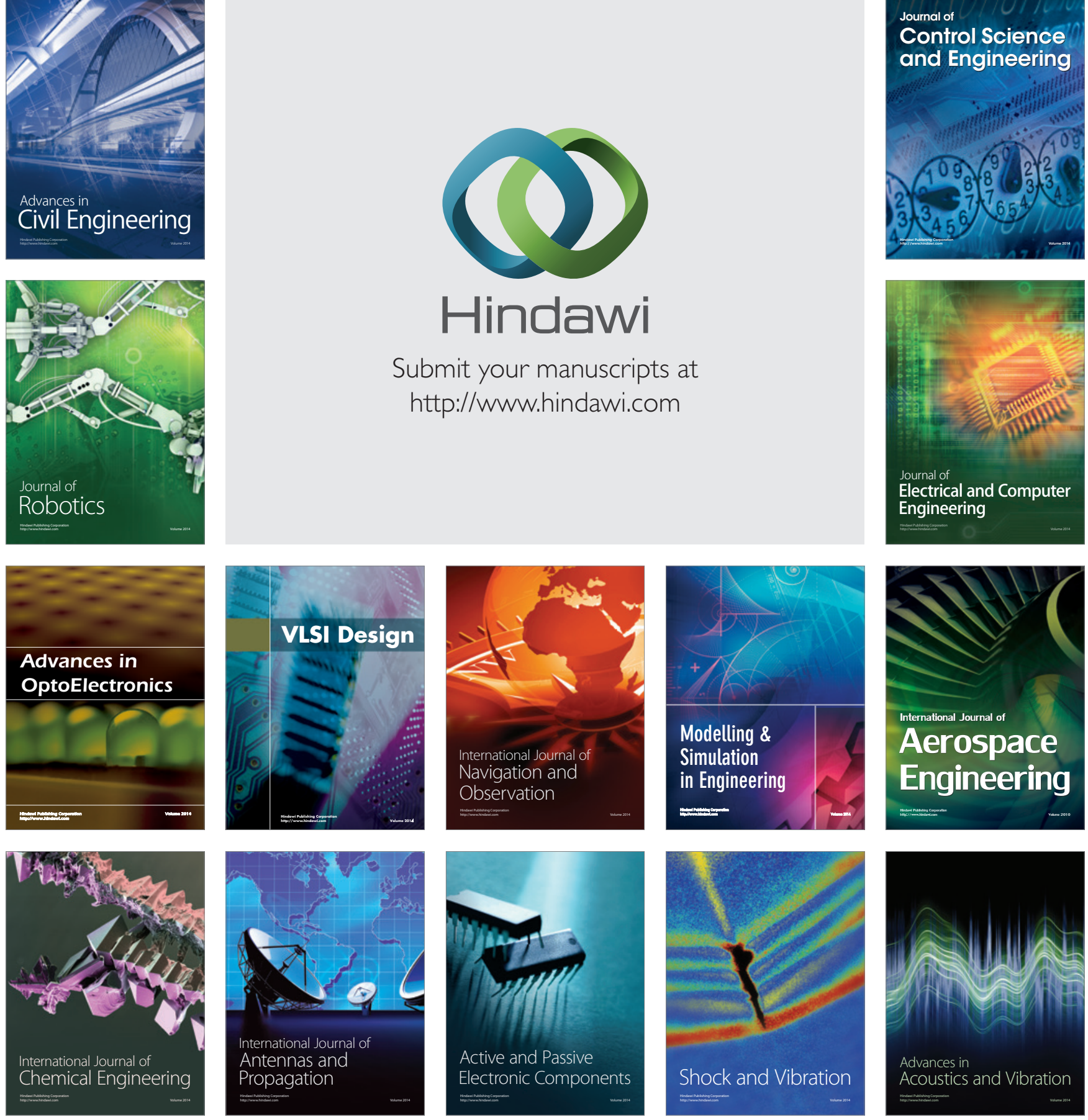